Meta

Journal des traducteurs

Translators' Journal

\title{
Terminologie et dictionnaires
}

\section{Teresa Cabré}

Volume 39, numéro 4, décembre 1994

Hommage à Bernard Quemada : termes et textes

URI : https://id.erudit.org/iderudit/002182ar

DOI : https://doi.org/10.7202/002182ar

Aller au sommaire du numéro

Éditeur(s)

Les Presses de l'Université de Montréal

ISSN

0026-0452 (imprimé)

1492-1421 (numérique)

Découvrir la revue

Citer cet article

Cabré, M. T. (1994). Terminologie et dictionnaires. Meta, 39(4), 589-597.

https://doi.org/10.7202/002182ar

\section{Résumé de l'article}

Les problèmes associés à l'inclusion de la terminologie dans un dictionnaire normatif sont illustrés par les travaux menés actuellement à l'Institut d'Estudis Catalans. La spécificité d'un dictionnaire normatif pose à la terminologie le problème de sa propre "normati-vité ", qui est examiné sous l'angle linguistique et sous l'angle organisationnel, en tenant compte en particulier des domaines spécialisés, des termes spécialisés par domaines, de la problématique de la définition et de la présentation des domaines. 


\title{
TERMINOLOGIE ET DICTIONNAIRES
}

\author{
M. TERESA CABRÉ \\ Université Pompeu Fabra, Barcelone, Espagne
}

\begin{abstract}
Résumé
Les problèmes associés à l'inclusion de la terminologie dans un dictionnaire normatif sont illustrés par les travaux menés actuellement à l'Institut d'Estudis Catalans. La spécificité d'un dictionnaire normatif pose à la terminologie le problème de sa propre "normativité", qui est examiné sous l'angle linguistique et sous l'angle organisationnel, en tenant compte en particulier des domaines spécialisés, des termes spécialisés par domaines, de la problématique de la définition et de la présentation des domaines.
\end{abstract}

\begin{abstract}
The problems associated with the inclusion of terminology in a normative dictionary are illustrated by the work in progress at the Institut d'Estudis Catalans. The particularity of a normative dictionary brings out the problem of terminology's own "normativity," which is examined from two angles: that of linguistics and that of organization. Particular attention is paid to special subject areas, to terms specialized by subject area, to the problem of the definition and the presentation of subject fields.
\end{abstract}

La linguistique descriptive [...] condamne les désignations de «langue» technique et scientifique qui sont également impropres. Il convient plutôt de parler de "vocabulaires», s'agissant d'emplois particuliers du français et de ses variétés, qui font appel, pour la prononciation, la morphologie et la syntaxe, au fonds de la langue commune, caractère à partir duquel notre analyse établit des rapports fonctionnels mais non hiérarchisés. (Quemada 1978: 1153)

L'entrée de termes dans un dictionnaire général est une question qui a été longuement et diversement débattue, elle n'a cependant pas encore fait l'objet d'une réflexion sérieuse et systématique.

Certes, c'est un fait que les dictionnaires de langue générale incluent de la terminologie spécialisée, un usager non averti peut aisément le remarquer en découvrant des mots renvoyant à des domaines de spécialité à travers des marques de spécialisation thématique. Pourtant, un grand nombre de questions sur le thème restent en suspens, plus spécialement celles qui font référence au concept même de terminologie, aux critères choisis pour sélectionner les termes devant être inclus dans un dictionnaire général ainsi qu'aux bases de l'organisation du travail terminologique du dictionnaire.

Notre article se veut une réflexion à propos de quelques-uns des points qui touchent à la sélection de la terminologie destinée à entrer dans un dictionnaire général. Pour des raisons de place, il nous sera impossible de traiter ici, en relation avec notre thème, les critères spécifiques qu'il a été décidé d'appliquer lors de l'actualisation du dictionnaire normatif de la langue catalane (Nou Diccionari General de la Llengua Catalana: NDGLC).

\section{UNE QUESTION DE PRINCIPE}

La terminologie dans un dictionnaire général doit correspondre à deux exigences. Premièrement, cela doit être la terminologie vraiment fondamentale. Le dictionnaire général doit enregistrer la terminologie dans les limites où elle est nécessaire - outre le domaine du travail 
professionnel - à un homme parlant la langue commune. Deuxièmement, dans les limites ainsi admises la terminologie doit être complète. (Walczak 1991: 129)

Ainsi donc personne ne met en doute, tel est le sens de la prise de position ferme de Walczak, la citation antérieure, que la terminologie doive faire partie du corps d'un dictionnaire général; de même, personne ne peut répondre concrètement et précisément à la question de savoir quelle terminologie doit entrer dans un dictionnaire général, ou quelle proportion de termes un dictionnaire doit contenir par rapport au nombre de mots qu'il englobe ou bien encore comment doit-on illustrer ces termes.

La réponse à toute cette série d'interrogations n'est, à la vérité, pas aussi simple que ce qu'on pourrait imaginer de prime abord. Elle oblige en effet à se poser toute une suite de questions à propos des concepts fondamentaux de la terminologie et de la lexicographie.

En premier lieu, il convient de préciser ce qu'on entend généralement par terminologie, et quelle est l'utilisation que nous en faisons dans notre travail.

Le terme terminologie est aujourd'hui une forme banalisée, tant par le manque de précision dans son emploi que par la confusion sur ses limites d'utilisation. Il convient d'emblée de préciser que le mot terminologie est en lui-même polysémique, comme l'ont déjà fait remarquer maintes publications générales sur la question; il sert tout autant à désigner la matière dont se charge l'étude ainsi que la compilation de termes, qu'à se référer à un ensemble de termes d'une discipline spécialisée. Dans sa seconde acception, le mot terme sert à désigner chacune des unités de cet ensemble.

Les termes, pour ce qui est des domaines de spécialité, sont des unités dans le sens littéral du mot: unités tout autant de forme que de signifié qui servent à se référer à la réalité spécialisée. L'ensemble des termes d'un champ, qui constitue la terminologie de ce champ, en reflète l'organisation conceptuelle. Chacun des termes représente un concept de la structure d'une matière.

Les utilisateurs habituels de la terminologie spécialisée, qui sont les spécialistes de chaque matière, emploient les termes dans les communications spécialisées dans un but principalement référentiel. Les linguistes, les lexicographes, les terminologues regroupent les termes propres à chaque matière dans les textes spécialisés produits par des techniciens, des scientifiques et, après leur avoir donné une forme lexicographique précise, ils les incluent dans les dictionnaires, les lexiques et les vocabulaires. Ainsi donc, la terminologie se trouve dans la documentation spécialisée dans son état naturel, ce n'est qu'après sa codification qu'elle apparaît dans les dictionnaires.

Les termes, tout comme les mots du lexique général, sont donc des unités de sens distinctives et significatives qui interviennent de façon naturelle dans le discours spécialisé pour se référer aux «objets» d'une réalité structurée.

Comme toute autre unité significative d'un système linguistique, les termes font partie d'un système structuré, à l'intérieur duquel ils occupent un niveau déterminé (le niveau des unités lexicales); en outre, d'une part, ils sont en relation avec le reste des unités du même niveau et d'autre part, avec les unités des autres niveaux, avec lesquelles ils participent à la construction du discours.

\section{TERMINOLOGIE ET LEXIQUE COMMUN}

The fact that humbler occupations like nursing, book-keeping and cooking and even hobbies also involve special areas of human interest and therefore also require and indeed have their own special language is much left often acknowledged. Since practically every human activity can be attributed to one subject or another, all language could be split into so many subject languages and the word "special" would be superfluous. (Sager, Dungworth, McDonald 1980:3) 
L'ensemble des mots spécialisés d'une discipline déterminée (également d'un domaine d'activité spécifique) constitue, on le sait, la terminologie propre de cette spécialité. Les termes, qui sont des unités de base de la terminologie, désignent les concepts propres à chaque discipline spécialisée.

En apparence, les termes ne semblent guère différer des mots si on les considère d'un point de vue formel ou sémantique; cependant, ils s'en différencient sensiblement si on les soumet à des critères de pragmatique et de communication. En effet, la particularité la plus notable de la terminologie, à la différence du lexique commun, réside dans le fait qu'elle sert à désigner des concepts propres aux disciplines et aux activités de spécialité. Par conséquent, les termes sont connus fondamentalement par des spécialistes de chacune des matières et ils apparaissent avec une fréquence plus élevée dans les documents spécialisés de chaque discipline. Les textes spécialisés se différencient des textes généraux par des aspects qui font référence à la terminologie, alors que la terminologie confère à ces textes des caractéristiques particulières. Pour ce qui est du contenu, l'appartenance des termes à des domaines concrets différencie évidemment les textes; de plus, le caractère monoréférentiel des termes joue un rôle clé dans la précision et l'univocité de la communication spécialisée.

Pour ce qui est de la forme, la concision de la terminologie (surtout s'il s'agit de création dans une langue et non d'une adaptation d'une dénomination étrangère), la fréquence d'apparition des formants savants et le caractère international des termes contribuent à l'aspect concis des textes spécialisés et favorisent la communication internationale, si importante pour les spécialistes.

Néanmoins, essayer d'établir une frontière nette entre les langues de spécialité et la langue commune est une tâche difficile. Il est certain que le problème fondamental de délimitation de la terminologie ou du lexique spécialisé en opposition avec le lexique commun repose sur la notion de spécialisé. En effet, on peut observer au premier abord que le mot spécialité couvre deux concepts de spécialisation : la spécialisation par la thématique, et la spécialisation par les caractéristiques de processus des échanges d'informations.

La première conception, qui représente le critère le plus répandu parmi les spécialistes, permet de classer les langages spécialisés selon les matières et les sous-matières spécifiques (physique, chimie, ingénierie électrique, anatomie, anatomie du système nerveux, etc.). Les matières scientifiques comme les expérimentales, les exactes, les matières humanistes ou les écomomiques et les juridiques, les techniques comme les spécialités du génie, la construction ou les communications, les domaines spécialisés d'activités comme le sport, le commerce ou les finances, génèrent des types de textes qui divergent sur quelques points des types communicatifs jugés comme habituels et propres à la langue commune.

Ainsi, cependant, il est malaisé de déterminer seulement par la thématique quels textes doivent être considérés comme des textes de spécialité et quels autres doivent être écartés et cela pour au moins deux raisons :

a) parce que la vie de tous les jours est pleine de domaines spécialisés à un certain degré;

b) parce qu'il se produit un mouvement permanent de termes du lexique général au lexique de spécialité, du lexique des spécialités au lexique commun, et des lexiques des spécialités entre eux.

Selon le second critère, la notion de spécialité couvre les différentes circonstances du discours conçu comme un processus de communication impersonnelle (caractéristiques des interlocuteurs, circonstances de communication, sujets de la communication, etc.). Ce second critère est moins vaste que le critère thématique à l'heure d'établir le 
concept de langage de spécialité et il ouvre la porte à beaucoup de circonstances par le seul fait qu'il ne suit pas le modèle de la communication standard (oraux spontanés, de thématique connue de n'importe quel locuteur d'une langue, moyennement formel, avec pour objectif principal l'élaboration de l'information ou son échange).

Du point de vue de la thématique, ne sont pas spécialisés les termes qui seraient matière de conversation et d'échange linguistique habituel dans les situations générales de la vie quotidienne des individus, thèmes qui ne demandent pas l'apprentissage d'un vocabulaire spécifique de la part des locuteurs. Du point de vue des interlocuteurs, tous les locuteurs ne sont pas des sujets spéciaux si l'on fait abstraction de leurs particularités dialectales les plus marquées ainsi que de leur appartenance à des groupes sociaux ou professionnels. Du point de vue des situations de communication, les situations communes à la majorité des membres d'une communauté sociale ne sont pas des situations «spéciales». Du point de vue fonctionnel, les communications informatives, avec une fonction principalement référentielle ne sont pas spéciales. Enfin, du point de vue du canal par lequel transite l'information, le modèle standard est constitué des communications orales, ou bien alors des communications écrites qui reproduisent les communications orales.

Un concept de spécialisation aussi large que celui que nous venons d'exposer rend extrêmement difficile la possibilité de cerner l'espace conceptuel de travail pour entreprendre une recherche. Il est donc inopérant.

En revanche, si la thématique est l'unique élément pris en compte pour délimiter le concept de langage spécialisé, il est difficile de décider quels sont les thèmes spécialisés et quels sont ceux qui ne le sont pas. Il paraît, en effet, évident que toute activité humaine demande une certaine spécialisation. Pour Sager, les langages spécialisés n'apparaissent pas dans la communication entre spécialistes.

Special subjects, or more precisely special subject languages are usually thought of as the means of expression of highly qualified subject specialists like engineers, physiciens, lawyers, etc. and are often derogatively referred to as "jargon." (Sager $1980: 3$ )

Cependant, tous les spécialistes ne partagent pas le critère restrictif de Sager. Pour Picht et Draskau, l'idiosyncrasie professionnelle des interlocuteurs est un facteur non pertinent, ils font, en revanche, une distinction entre les différents niveaux de spécialisation au sein de chaque langue de spécialité. Le degré de spécialisation le plus élevé correspond à la communication entre spécialistes, et le plus bas aux communications de caractère divulgatoire destinées au grand public.

It may not be true to say, with Sager et al., that "the use of LSP presupposes special education and is restricted to communication among specialists in the same or closely related fields ;" communication between experts is, as we have seen, only one of the higher levels at which LSP may be used. LSP is also used for the purposes of initiation and instruction, training and development at a lower level of abstraction and specialization. (Picht et Draskau $1985: 21$ )

Dans notre travail, nous concevons les langages de spécialité ou les langages spécialisés comme des sous-groupes du langage général, caractérisés pragmatiquement par trois variables : la thématique, les utilisateurs et les situations de communication, qui répondent aux présupposés suivants :

1) Nous considérons comme thématiques spécialisées celles qui font partie de la connaissance générale des locuteurs d'une langue et qui ont fait l'objet d'un apprentissage particulier.

2) Nous considérons que les utilisateurs types des langages de spécialité sont les spécialistes. Sur ce point, pourtant, nous faisons une différence entre les producteurs de 
communications spécialisées et leurs récepteurs. Seuls peuvent être producteurs de communications technico-scientifiques ou professionnelles de niveau restreint ceux qui possèdent une connaissance spécifique de la thématique, connaissance acquise par l'apprentissage. Peuvent en être considérés récepteurs tout aussi bien les spécialistes d'un thème, qui peuvent alors agir interactivement comme émetteurs dans n'importe quel acte de communication, que le public en général, qui reçoit passivement en qualité d'apprenant les communications spécialisées.

3) Nous pensons que les situations de communication déterminent également le caractère spécialisé d'un sous-code, par le fait que ses communications sont de type formel, normalement régies par des critères professionnels ou scientifiques.

4) Un langage de spécialité possède un certain degré de variation interne, en raison principalement de deux paramètres : le degré d'abstraction et les sujets de communication.

5) Les langages de spécialité sont en relation d'inclusion par rapport au langage général et en relation d'intersection avec la langue commune, avec laquelle ils partagent des caractéristiques et ils entretiennent une relation de flux constant d'unités et de conventions.

\section{TERMINOLOGIE ET LEXIQUE BANALISÉ}

L'ensemble de la terminologie des disciplines particulières de la science et de la technique est noté par les dictionnaires spéciaux uni-, bi- et multilingues. Dans les dictionnaires généraux la sélection est indispensable. En théorie on y enregistre la terminologie de baseles termes les plus importants, les plus répandus, les plus largement connus et employés. (Walczak 1991: 127)

Il est reconnu que chaque matière spécialisée, selon les concepts que nous avons établis jusqu'à présent, dispose d'une terminologie propre. Cependant, cette affirmation apparemment si innocente cache en pratique une complexité importante. Bien sûr, entre un ensemble et un autre ensemble tout aussi bien de la langue générale que des langues de spécialité, se produisent perpétuellement des mouvements, ce qui fait que ce que l'on considère un temps spécialité puisse devenir commun peu de temps après, ce qui fait également qu'entre les diverses disciplines se produise un échange ininterrompu et constant de termes.

La grande extension des moyens de communication de masse et la démocratisation de l'enseignement ont favorisé cette divulgation des matières spécialisées, et, par conséquent, la diffusion de la terminologie la plus employée.

Des mots comme robotique, informatique, ordinateur, micro-ordinateur, microinformatique ou traitement de texte font partie de la terminologie générale d'un locuteur moyennement informé qui l'ignorait totalement voici dix ou vingt ans. D'autres mots comme animatique, bureautique, domotique ou constructique, selon le rythme de développement des progès technologiques et de leur diffusion commerciale, seront connus par le même type de locuteurs.

Parallèlement, un échange important se produit également de la langue commune vers les langues spécialisées. Ce phénomène repose sur la terminologisation des mots de la langue générale, qui passent à divers domaines avec des signifiés précis dans chacun d'eux, et, par conséquent, différents entre eux. Les termes provenant du corps humain en sont un bon exemple (bras, tête, membre, corps, pied, ail, cerveau, etc.) qui sont à l'heure actuelle utilisés avec un signifié propre en mécanique, en construction, en administration, en géologie, en informatique, en urbanisme, etc. Pour cette raison, la distinction entre «appartenir à un champ spécialisé» et «être utilisé dans un champ spécialisé» est de plus en plus généralement acceptée. Un terme appartient seulement à un champ spécialisé; il peut appartenir à divers sous-domaines de ce champ principal, ou bien alors il peut être 
utilisé dans nombre d'autres champs de spécialité. Par exemple, variable est un terme de mathématiques employé en statistique et en économie, virus est un terme de médecine réadapté par l'informatique, cellule, qui est un terme de cytologie, est passé à l'électronique: 'cellule solaire', 'cellule photoélectrique', 'cellule voltaïque'. Le terme grammaire («ensemble ordonné de règles grammaticales») est une unité qui appartient à la linguistique, bien qu'elle soit également employée dans le commerce de l'édition, avec une perspective bien différente (type de livre qui possède des caractéristiques et des acheteurs déterminés), de même, le terme écologie et ses dérivés ont été utilisés dans le discours public politique et sociologique avec des valeurs et des connotations bien différentes de celles qu'ils connaissaient dans le milieu strictement scientifique.

La réalité est un continuum qui est segmenté seulement par artifice en matières, et tout phénomène scientifique peut être analysé à partir de maints points de vue divers et à partir de perspectives scientifiques différentes. Cette multilatéralité de la science empêche que l'on puisse établir une frontière nette entre une matière scientifique et une autre et, par conséquent, entre les frontières de la terminologie de chaque matière. Tel est le cas de termes comme vecteur (qui appartient aux mathématiques et à la physique) ou ton (qui peut être défini à partir de la musique ou de l'acoustique). Un même terme, enfin, avec son concept dominant, peut être utilisé dans divers buts, et, par conséquent, par divers utilisateurs. La perspective d'un usager professionnel d'une matière peut générer une terminologie spécialisée.

\section{LEXICOGRAPHIE ET TERMINOLOGIE}

Afin de saisir la langue tout entière dans sa diversité, on a trouvé utile de la découper en sous-systèmes plus homogènes. L'un des découpages possibles c'est la division «externe» mais fructueuse de l'ensemble de la langue en plusieurs (sous-)langues dites fonctionnelles. (Kocourek 1982: 14)

L'élaboration d'un dictionnaire est un procès en diverses phases pour chacune desquelles l'auteur effectue une série de choix qui le conduisent en fin de compte à des types de dictionnaires différents. Les recueils terminologiques se différencient en ce point des recueils lexicaux en ce que, durant quelques phases du processus, on sélectionne des possibilités déterminées et on se démarque d'autres, qui sont plus propres aux dictionnaires de lexique général. Ainsi donc, en terminologie, on utilise la documentation spécialisée, orale ou écrite, comme unique source de matériel; on sélectionne les entrées, qui sont toujours lexématiques (bien que composées d'un ou de plusieurs mots), en fonction de la thématique, on conserve uniquement les informations considérées comme pertinentes en terminologie, et on présente ces informations - surtout dans le domaine de la terminologie technico-scientifique - en suivant le plus possible la norme internationale.

Dans le projet de n'importe quel dictionnaire général, cependant, la terminologie se doit d'occuper une place importante, si ce dictionnaire se propose d'être au service des utilisateurs d'une langue. En effet, nous partons de la base qu'un dictionnaire doit tenir compte de la compétence moyenne du locuteur cultivé, mais il est évident que ce locuteur connaît une série d'unités lexicales liées aux spécialités. Un dictionnaire général doit donc intégrer ces termes dans l'ensemble de sa nomenclature.

La problématique repose, cependant, sur le fait de savoir sur quels critères sélectionner la terminologie qui doit être incluse dans un dictionnaire général et sur la façon de présenter ces termes spécialisés dans un dictionnaire de langue.

Pour apporter une réponse à la première question, il faut préciser avant toute chose ce que nous considérons être un langage dont fait partie la terminologie. La majorité des spécialistes s'accordent à classer les langages spécialisés selon deux axes: l'un, vertical, 
basé sur la thématique, l'autre, horizontal, basé sur le style et le degré d'abstraction à partir duquel on peut présenter un contenu.

Le premier axe permet de distinguer les différents langages de spécialité classés par blocs de matières, par matières, par sous-matières, etc. C'est la base de quelques classes de marques pragmatiques que l'on utilise dans un dictionnaire général. Du point de vue thématique, les divers langages de spécialité sont un continuum dans lequel on peut distinguer les diverses aires thématiques, chacune d'elles constitue un langage de spécialité déterminé. Non seulement les sciences et les techniques traditionnelles feraient partie de cette classification, mais aussi les domaines spécialisés de la vie professionnelle comme la gestion, la production et la consommation. Toutes les aires thématiques peuvent être, en principe, l'objet de la connaissance d'un locuteur cultivé, tout au moins à certains degrés de profondeur.

Le second axe de classement des langages de spécialité établit que dans chaque langue, il est possible de distinguer divers degrés d'abstraction qui obligent à l'établissement de divers styles discursifs selon le type de situation de communication auquel il faut apporter une réponse. La terminologie sert à dénommer une réalité spécialisée propre à une science, à une technique ou, en un sens plus large, à une activité restreinte, et une unité acquiert simplement le statut de terme en étant située dans la structure d'un champ de spécialité. Malgré ces faits, la banalisation tant de la spécialité en elle-même (aires spécialisées de la vie quotidienne des individus), que celle des termes proprement technicoscientifiques, fait que le locuteur moyen de la langue connait une gamme plus étendue de termes de spécialité, qui pourraient être distribués en deux groupes : a) les termes spécialisés des domaines de la vie quotidienne, qui englobent également les matières spécialisées du point de vue de la consommation (sport, gastronomie, loisirs, esthétique, bricolage, etc.), b) les termes proprement spécialisés qui forment le tronc commun des matières scientificotechniques, des matières de gestion et de production.

Par conséquent, on pourrait dire que la terminologie proprement dite est le véhicule de la communication formelle professionnelle des spécialistes de ce domaine, mais que les locuteurs moyennement érudits d'une langue connaissent activement un bon nombre de termes de spécialisation moyenne et connaissent passivement (principalement à travers la diffusion dont ils sont l'objet de la part des moyens de communication) un plus grand nombre de termes proprement considérés comme termes de spécialité. Ces termes sont ceux que doit recueillir un dictionnaire général de langue qui se propose d'être d'une utilisation efficace pour les usagers.

\section{LEXICOGRAPHIE ET TERMINOGRAPHIE}

Le déroulement du travail en terminologie (la terminographie) conduit à un même point que celui de la lexicographie: la confection de dictionnaires, les deux matières en application divergent par des aspects qui font qu'un dictionnaire de lexique commun soit un produit différent d'une terminologie spécialisée.

En effet, les recueils terminologiques se différencient des recueils lexicaux précisément par le déroulement même du travail et par les finalités que ce déroulement se propose dans chaque cas.

En terminologie, on se fonde sur la documentation spécialisée (orale ou écrite) d'un domaine précis comme source exclusive de recherche des termes, on sélectionne les entrées en fonction de leur pertinence par rapport à un domaine de spécialité, on les représente toujours sous leur forme lexématique et on les illustre seulement en apportant ces informations que l'on considère pertinentes dans un seul champ de spécialité qui est l'objet du dictionnaire.

Les termes spécialisés que comprend un dictionnaire général, cependant, ne peuvent pas être observés à partir de la vision précise de la terminologie, mais ils sont plutôt vus 
comme les unités lexicales du système de la langue et, par conséquent, propres à un code lexical d'un locuteur moyennement cultivé.

Si cela est la focalisation à partir de laquelle le lexicographe doit travailler, les unités terminologiques doivent être traitées dans le dictionnaire comme des mots de la langue dans son acception la plus forte et, par conséquent, il faut les présenter comme on présente les autres mots.

La restriction quant à leur origine (qui indique un usage éventuellement restreint à un certain domaine) est représentée ordinairement à travers un ensemble d'étiquettes thématiques contrôlées systématiquement par le lexicographe. La définition qui les accompagne se limite à représenter le contenu du mot avec son domaine restreint. Cependant, le type de définition qui accompagne le terme dans une terminologie ne peut être en aucune manière celui qui spécifie le signifié du terme dans un dictionnaire général, tout au moins pour deux raisons fondamentales:

a) parce que l'usager type est différent: dans le cas de la terminologie, c'est le spécialiste qui connaît la structure conceptuelle de la matière à laquelle appartient le terme, dans le cas du dictionnaire général, c'est le locuteur de la langue qui peut méconnaître cette structuration;

b) parce que la perception de la réalité de la part du spécialiste ne coïncide pas avec celle du locuteur commun et, par conséquent, la définition des termes dans un dictionnaire général doit s'accorder principalement avec les caractéristiques privilégiées par les locuteurs de la langue. Ceux-ci ne conceptualisent pas les mots dans un domaine restreint à une spécialité formellement structurée mais plutôt dans l'ensemble du système linguistique.

Au moment où les termes scientifiques entrent dans un dictionnaire général, ils deviennent des mots au sens fort, et ils doivent être traités en tant que mots par le lexicographe.

L'objectif prioritaire que se propose la terminologie est la normalisation (au sens de standardidation) des termes propres à un domaine spécialisé précis. Ainsi donc, on ne se limite pas à recueillir les dénominations d'une aire déterminée dans un but informatif ou descriptif, mais on agit avec l'objectif de fixer des unités terminologiques comme formes normalisées, en établissant les formes de référence, en écartant les autres variantes pour dénommer un même concept. On tente avant tout d'écarter les diverses possibilités de déterminer une autre réalité. Les dictionnaires généraux, y compris les normatifs (qui en lexicographie générale sont de par leur fonction l'unique type de dictionnaire qui converge avec la finalité de la terminologie), recueillent les mots qui sont utilisés dans une langue, dans toute sa diversité et sa richesse expressive. Chaque entrée de dictionnaire est une unité formelle avec diverses acceptions sémantiques selon le milieu dans lequel elle est utilisée, et c'est l'ensemble des différentes acceptions qui constitue l'information de chaque mot.

\section{RÉFÉRENCES}

Actas del colóquio de lexicologia e lexicografia (1990) : Lisboa, Universidade Nova de Lisboa.

BÉJOINT, Henri (1988): «Scientific and Technical Words in General Dictionaries», International Journal of Lexicography, 4, pp. 354-368.

CABRÉ, M. T. (1991): «Terminologie ou terminologies? Spécialité linguistique ou domaine interdisciplinaire?», Meta, 36-1.

CABRÉ, M. T. (1992) : La terminologia. Teoria, mètodes, aplicacions, Barcelona, Empúries.

CLAS, A. et al. (1985): Guide de recherche en lexicographie et terminologie, Paris, Agence de Coopération Culturelle et Technique.

CORBIN, D. et P. CORBIN (1980): «Le monde étrange des dictionnaires (1): Les marques d'usage dans le Micro-Robert», Synonymies, 4. 

GAMBIER, Y. (1991): «Présupposés de la terminologie : vers une remise en cause», Cahiers de linguistique
sociale, 18, pp. 31-58.

GUILBERT, L. (1977) : «Lexicographie et terminologie», Terminologies 76.

KOCOUREK, R. (1982): La langue française de la technique et de la science, Wiesbaden, Brandstetter.

QUEMADA, B. (1978) : «Technique et langage», Histoire des techniques, Encyclopédie de la Pléiade,

QUEMADA, B. (1987) : «Notes sur lexicographie et dictionnairique», Cahiers de lexicologie, 51, 2

REY, A. (1988): «Terminologie et lexicographie», Paralleles, 10, pp. 27-35.

SAGER, J. C., DUNGWORTH, D. and P. F. McDONALD (1980):

Practice in Science and Technology, Wiesbaden, Oscar Brandstetter. ALSED-LSP Newsletter, 9,2(23).

WALCZAK, O. (1991) : «La terminologie dans les dictionnaires généraux», Neoterm, 13-16, pp. 126-129. 